\title{
Surgical Management and Scheimpflug Analysis of an Atypical Lens Coloboma
}

\author{
Julio C. Hernadez-Camarena ${ }^{b}$ Eduardo Ayup-Arguijo ${ }^{a}$ \\ Eduardo Chavez-Mondragon $^{a}$ Arturo Ramirez-Miranda ${ }^{a, b}$ \\ ${ }^{a}$ Anterior Segment Department and ${ }^{b}$ Cornea and Refractive Surgery \\ Department, Instituto de Oftalmología Fundación Conde de Valenciana, \\ Mexico City, Mexico
}

\section{Key Words}

Lens coloboma - Capsular tension ring - Scheimpflug image

\begin{abstract}
A lens coloboma is not a true coloboma; it is just a zonular absence that causes a defect in the lens equator and hence a more spherical lens. It can be isolated or in association with iris, choroid or retinal colobomas. Typically the defect is present at the site of the embryonic fissure, but it can be present elsewhere. This congenital defect can cause amblyopia, especially when it is associated with cataract. We describe a case of a 39-year-old male with an atypical coloboma that was managed successfully with phacoemulsification, capsular tension ring and intraocular lens implantation. Scheimpflug image analysis and full ophthalmological assessment were performed. Scheimpflug images demonstrated a notching of the lens equator and an absence of zonulae. During the surgical procedure an ophthalmic viscosurgical device was used to tamponade the vitreous, and after continuous curvilinear capsulorhexis the capsular tension ring was inserted to allow the surgeon to perform a safe phacoemulsification.
\end{abstract}

\section{Introduction}

A lens coloboma is the result of a segmentally defective or absent development of the zonulae, giving the clinical appearance of flattening or notching of the lens equator in the region of zonular defect [1]. Therefore, lens coloboma is not a real coloboma, since there is not an actual loss of tissue; it consists of a thicker and more spherical lens as a consequence of the unequal pull of the zonular fibers [2]. This anomaly typically present in the inferior-nasal quadrant (at the site of the embryonic fissure) and can 
occur as an isolated malformation in one eye or bilaterally associated with uveal colobomas [3].

\section{Case Report}

A 39-year-old male with no relevant medical or ophthalmologic history presented to an ophthalmologic routine exam following long-standing blurred vision and a 'white eye' appearance in his left eye. The uncorrected distance visual acuity (UDVA) was 20/15 in his right eye and 3/200 in the left eye that corrected to $20 / 300$ with a manifest refraction of $-7.50=-3.75 \times 175$. The slit lamp exam under mydriasis revealed a lens coloboma in the superior-nasal quadrant and a nuclear congenital cataract (fig. 1a, b). Fundus exam was unremarkable in the right eye and the left eye showed choroidal appearance and peripapillary atrophy. The optic biometry with the IOL Master (Carl Zeiss Meditec, Germany) showed an axial length of $23.14 \mathrm{~mm}$ in the right eye and $28.85 \mathrm{~mm}$ in the left eye, with keratometry readings of $46.00 / 47.00 \times 180$ and $45.49 / 49.93 \times 180$, respectively. The SRK-T formula predicted an IOL power of $-1.00 \mathrm{dpt}$ to obtain emmetropy. Scheimpflug images obtained with the Pentacam HR (Oculus, Germany) were used to analyze the morphology of the lens coloboma (fig, $2 \mathrm{a}$, b). The patient underwent phacoemulsification in the left eye using a capsular tension ring and an IOL (ACTR12 and Acrysof MA60MA; Alcon, Fort Worth, Tex., USA) with a dioptric power of +1.00 (fig. 3 ). During surgery vitreous loss through the colobomatous region was observed, requiring automated anterior vitrectomy. Eight days after the surgery, the patient was asymptomatic and referred a notable visual acuity improvement on his left eye, showing a UDVA of 20/40 and a corrected distance visual acuity (CDVA) of 20/30 with a manifest refraction of $+0.25=-3.50 \times 102$. On slit lamp examination a well-centered and stable IOL was observed in the capsular bag with no evidence of vitreous in the anterior chamber. Postoperative Scheimpflug images were obtained (fig. $\underline{4} a-c)$.

\section{Discussion}

Lens coloboma is a rare idiopathic congenital disorder characterized by a notching of the crystalline lens due to corresponding zonular absence or weakness [2, 4]. It can be either isolated or can occur associated with other ocular or systemic malformations [5]. Typical lens colobomas are located inferonasally, consistent with a defect on the embryonic fissure closure. The embryologic genesis of atypical colobomas, as the case we present here, is uncertain; a number of theories propose a rotation of the embryonic fissure, inflammatory process or persistence of vascular mesodermal tissue that blocks the forward growth of the neuroectoderm $[3,6]$. Lens colobomas have been associated with astigmatic refractive errors and other ocular malformations [2], such as in our case, who presented a -3.75 refractive cylinder and a nuclear congenital cataract, which may have induced a form deprivation myopia [7] and would explain both the axial length difference and myopic features in the fundoscopy.

The surgical management of a lens coloboma is frequently defying since there is a high risk of capsular fornix aspiration, zonular dialysis extension, vitreous herniation into the anterior chamber, IOL decentration and closure of the capsular opening [8]. Hence, the implantation of a capsular tension ring is preferred before the emulsification of the nucleus to prevent capsular bag deformity, enable nucleus rotation and avoid capsular collapse; therefore, reducing surgical complications [9]. In order to enhance surgical planning, a detailed imaging of the lens, zonulae and ciliary body is needed. High-resolution ultrasound biomicroscopy has been suited before for in vivo study of the human zonular apparatus and lens [1]. We suggest the use of Scheimpflug images obtained with the Pentacam HR (Oculus, Germany) to depict a more accurate view of 
the lens coloboma morphology and to anticipate the potential complications during surgery. In accordance with previous reports [1, 9], we found an augmented sphericity of the lens and zonular absence at the region of the coloboma; sustaining the theory that lens coloboma results rather from the faulty mechanical tension of the zonulae than from a primary defect on the lens. Scheimpflug images showed to be a great ancillary imaging study to explore lens coloboma, to plan its surgery and to elucidate its pathology.

\section{Disclosure Statement}

Presented as a poster on the 2nd International Congress of Cataract Surgery, Phaco-Refractive Surgery and Intraocular Lenses, Acapulco, Guerrero, México, August 2007, and on the Annual Meeting of the American Academy of Ophthalmology, Atlanta, Ga., USA, November 2008. No author has a financial or proprietary interest in any material or method mentioned.
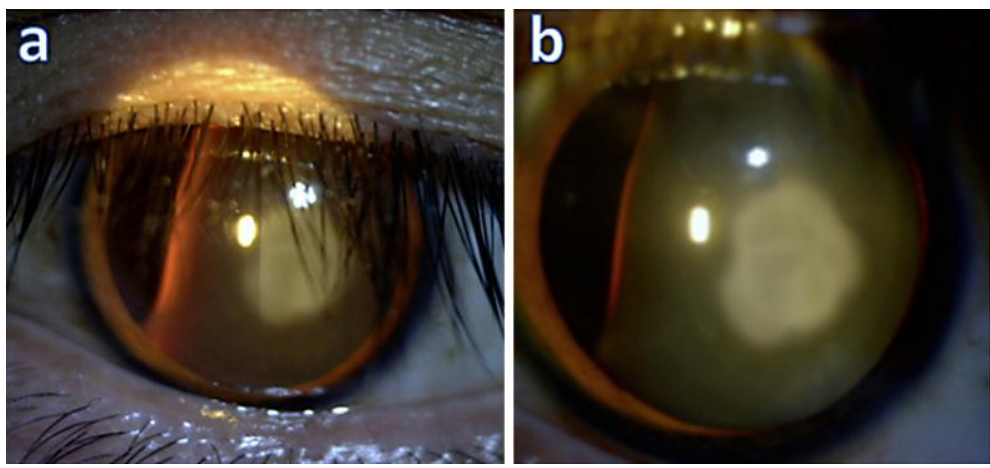

Fig. 1. a Clinical photograph shows temporal notch, sphericity loss of the crystalline lens, and nuclear cataract involving visual axis. $\mathbf{b}$ Close up of the lens colobomatous area, and intact lens capsule is appreciated.
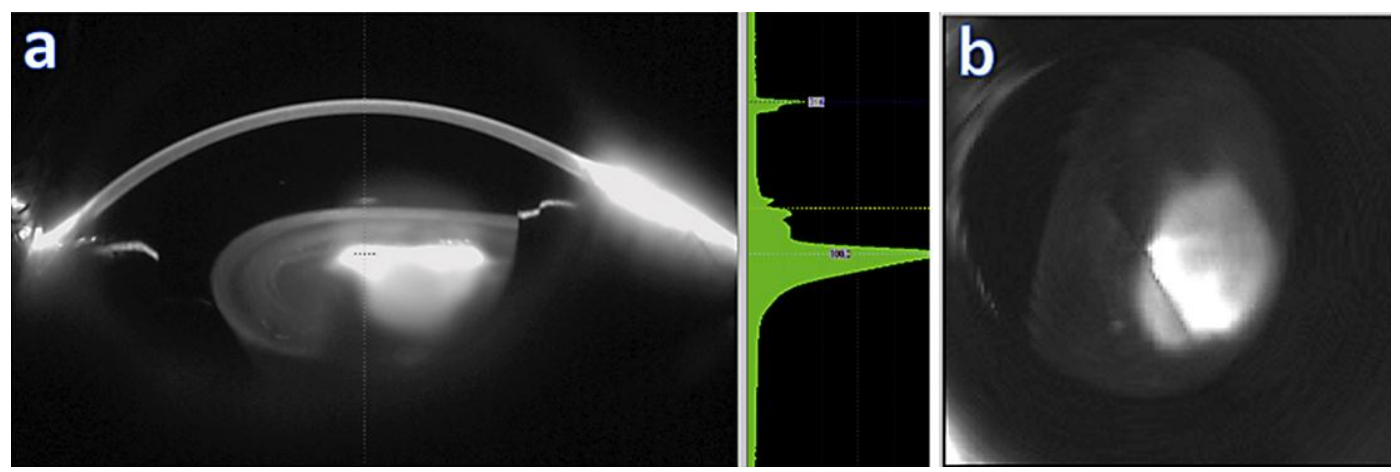

Fig. 2. a Scheimpflug images show the absence of zonules in the temporal region, generating change in lens sphericity and a decrease in its equatorial length. Lens densitometry shows opacity with a density of 100, compared with a density of 0 for aqueous humor and 25-35 for clear cornea and lens. b Ocular tomography in a coronal cut of the left eye showing the temporal lens coloboma and a central lens opacity. 


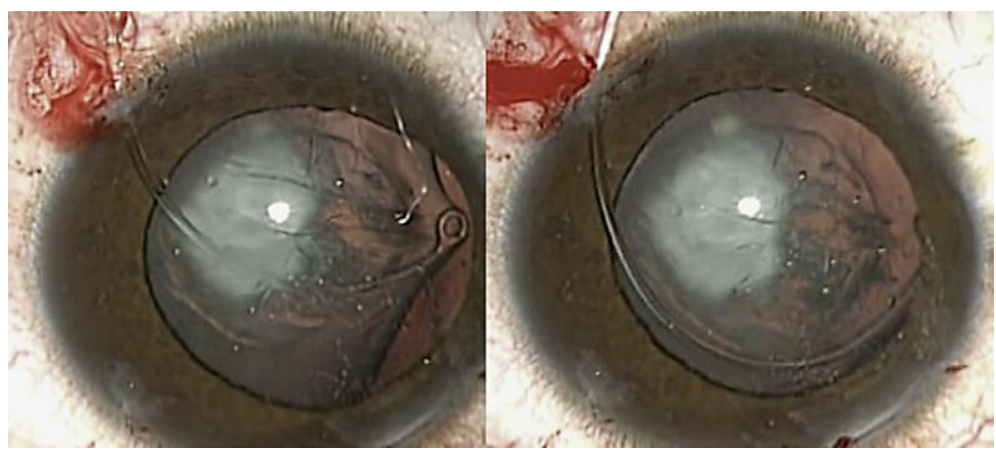

Fig. 3. Intraoperative image at the moment of insertion of the capsular tension ring, showing stabilization of the capsular bag and correction of the colobomatous area.
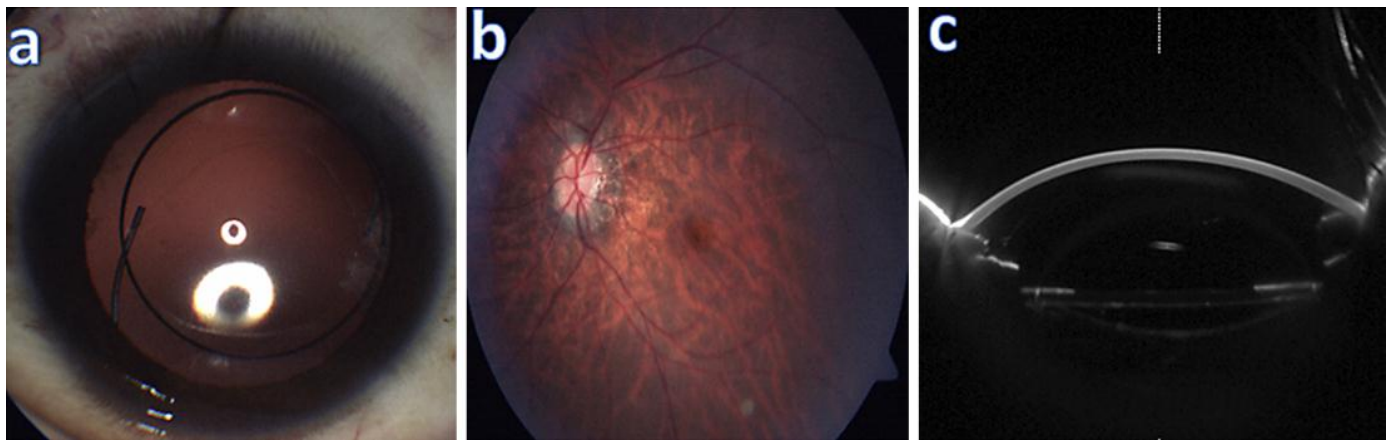

Fig. 4. a Postoperative clinical photograph showing a stable capsular bag with a centered IOL, and no vitreous strands. b Fundus photograph of the left eye, showing choroidal fundus with normal characteristics and peripapillary atrophy and healthy macula. c Scheimpflug image of the patient one week after the surgical procedure, a weakly formed capsular bag and IOL is observed.

\section{References}

1 Agarwal T, Saxena R, Vajpayee RB: Ultrasound biomicroscopy in lens 'coloboma'. Eur J Ophthalmol 2003;13:390-391.

-2 Bavbek T, Ogut MS, Kazokoglu H: Congenital lens coloboma and associated pathologies. Doc Ophthalmol 1993;83:313-322.

-3 Onwochei BC, Simon JW, Bateman JB, Couture KC, Mir E: Ocular colobomata. Surv Ophthalmol 2000;45:175-194.

4 Khan A0, Al-Assiri A: Lens coloboma associated with a ciliary body cyst. Ophthalmic Genet 2007;28:208-209.

5 Ilsen PF, Patel S: Coloboma of the crystalline lens. Optometry 2003;74:765-774.

-6 Reis LM, Semina EV: Genetics of anterior segment dysgenesis disorders. Curr Opin Ophthalmol 2011;22:314-324.

7 Huo L, Cui D, Yang X, Wan W, Liao R, Trier K, Zeng J: A retrospective study: form-deprivation myopia in unilateral congenital ptosis. Clin Exp Optom 2012;95:404-409.

-8 Menapace R, Findl 0, Georgopoulos M, Rainer G, Vass C, Schmetterer K: The capsular tension ring: designs, applications, and techniques. J Cataract Refract Surg 2000;26:898-912.

-9 Mizuno H, Yamada J, Nishiura M, Takahashi H, Hino Y, Miyatani H: Capsular tension ring use in a patient with congenital coloboma of the lens. J Cataract Refract Surg 2004;30:503-506. 